\section{What Is Wrong With Our Schools of Public Health?}

Within a matter of weeks of each other, the heads of the Schools of Public Health (SPH) at the University of Alberta and the University of Montreal quit their jobs in 2011. The director of the SPH at the University of Toronto also left his job abruptly about a year earlier. Each had completed less than half of their first term. The public health $(\mathrm{PH})$ community is justified to worry about the consequences of those departures and ask what this might mean for the future of $\mathrm{PH}$ in Canada.

SPH play an essential role in the production, teaching and dissemination of knowledge for $\mathrm{PH}$. Graduate training programs, innovation in theory, research, knowledge exchange and engagement with key partners are critical roles of SPH to support an effective $\mathrm{PH}$ system. However, SPH are very young in this country and have not yet contributed significantly as organizations to the renewal or the strengthening of the $\mathrm{PH}$ system. SPH can be traced back to the Schools of Hygiene created at the beginning of the $\mathrm{XX}^{\text {th }}$ century at the University of Toronto and the University of Montreal. These were eventually merged into Faculties of Medicine as Departments of Social and Preventive Medicine in the 1970s. Since then, PH has mostly resided in Faculties of Medicine where, at best, it suffered from benign neglect or indifference from Faculty leadership. The renewed interest in $\mathrm{PH}$ was probably brought about by the early 2000s emergencies as well as by the prospect of attracting new funds from the recently created CIHR Institute of Population and Public Health and the Public Health Agency of Canada.

There are many lessons from the recent events. First, even the best of plans to introduce major university changes in an environment of constrained resources can be derailed easily. Second, not all Faculty members within SPH will be supportive of change because it may threaten existing advantages or privileges. Third, other Faculties and Departments are competing for the same limited resources and can be fierce opponents of change. Fourth, the vision of and a commitment to the SPH must be shared at the highest level of the University and the chief executives must exert enlightened leadership.

Will our universities step up to the plate and play their role in $\mathrm{PH}$ ? Will they invest the financial and human resources required to create outstanding SPH? Although new SPH are sprouting up all over the country, I doubt a deeply held belief in PH or a true commitment is widespread at the top. Only time will tell.

Gilles Paradis

Scientific Editor

\section{Qu'est-ce qui cloche avec nos écoles de santé publique?}

À quelques semaines l'un de l'autre, les dirigeants des écoles de santé publique (ÉSP) de l'Université de l'Alberta et de l'Université de Montréal ont démissionné en 2011. Environ un an plus tôt, le directeur de l'ÉSP de l'Université de Toronto avait lui aussi quitté son emploi brusquement. Chacun de ces responsables avait fait moins de la moitié de son premier mandat. La communauté de la santé publique est en droit de s'inquiéter des conséquences de ces départs et de leur signification pour l'avenir de la santé publique au Canada.

Les ÉSP jouent un rôle essentiel dans la production, l'enseignement et la diffusion des connaissances en santé publique. Les programmes de troisième cycle, les innovations théoriques, la recherche, l'échange des connaissances et la mobilisation de partenaires clés sont les rôles névralgiques que jouent les ÉSP pour renforcer l'efficacité du réseau de la santé publique. Cependant, les ÉSP sont très jeunes au Canada; elles n'ont pas encore contribué de façon appréciable, en tant qu'organismes, au renouvellement ni au renforcement du réseau. Les ÉSP trouvent leur origine dans les écoles d'hygiène créées au début du $\mathrm{xx}^{\mathrm{e}}$ siècle à l'Université de Toronto et à l'Université de Montréal. Ces écoles ont fini par être happées par les facultés de médecine, où elles sont devenues les départements de médecine sociale et préventive dans les années 1970. Depuis, la santé publique réside principalement dans les facultés de médecine, où au mieux, elle subit la négligence bienveillante ou l'indifférence des dirigeants de la faculté. Le regain d'intérêt pour la santé publique s'explique sans doute par les urgences sanitaires du début des années 2000 et par la perspective d'attirer de l'argent frais de deux institutions créées récemment : l'Institut de la santé publique et des populations des IRSC et l'Agence de la santé publique du Canada.

Il y a de nombreuses leçons à tirer des derniers événements. Premièrement, dans un climat de rareté des ressources, même les meilleurs plans pour introduire des changements universitaires majeurs peuvent facilement dérailler. Deuxièmement, tous les membres du corps professoral d'une ÉSP ne sont pas favorables au changement, qui peut menacer des intérêts ou des privilèges acquis. Troisièmement, d'autres facultés et départements se disputent les mêmes ressources limitées et peuvent s'opposer férocement au changement. Quatrièmement, la vision de l'ÉSP et l'engagement envers la nouvelle institution doivent être partagés au plus haut niveau de l'université, et les instances dirigeantes doivent exercer un leadership éclairé.

Nos universités prendront-elles leurs responsabilités et jouerontelles leur rôle en santé publique? Y investiront-elles les ressources humaines et financières requises pour créer des ÉSP exceptionnelles? Il pousse de nouvelles ÉSP dans tout le pays, mais je doute qu'au sommet, on soit pleinement convaincu de la valeur de la santé publique ou véritablement résolu à l'appuyer. Seul l'avenir nous le dira.

Le rédacteur scientifique, Gilles Paradis 